\title{
Effect of oral tizanidine on prolongation of intrathecal lidocaine
}

\author{
Arman Taheri', Morteza Hashemian², Mehrdad Noroozi2 ${ }^{2}$, Mohammad Bakhtiari ${ }^{3}$, Mohammad Reza \\ Doroodian $^{2}$, Shirin Salajegheh ${ }^{2}$
}

'Department of Anesthesiology, Tehran University of Medical Sciences, Tehran, Iran ${ }^{2}$ Department of Anesthesiology, Kerman University of Medical Sciences, Kerman, Iran

${ }^{3}$ Department of Anesthesiology, Sirjan School of Medicine, Sirjan, Iran

\begin{abstract}
Received: 11 March 2018
Accepted: 9 May 2018

Published online: 20 May 2018

*Corresponding author: Mehrdad Noroozi, Shahid Bahonar Hospital, Kerman Medical University, Kerman, Iran.

Email: mehrdadnoroozi48@yahoo. com

Competing interests: None.

Funding information: None.

Citation: Taheri A, Hashemian M Noroozi M, Mohammad Bakhtiari M Doroodian MR, Salajegheh S. Effect of oral tizanidine on prolongation of intrathecal lidocaine. Journal of Emergency Practice and Trauma 2018; 4(2): 62-66. doi: 10.15171/ jept.2018.06.
\end{abstract}

\begin{abstract}
Objective: In order to prolong the duration of intrathecal lidocaine, various drugs are used along with it. Due to the promising effects of tizanidine on central nervous system, it can be assumed that tizanidine can have a positive effect on increasing the anesthesia duration too. Thus, we aimed to investigate the effect of oral tizanidine on the duration of lidocaine spinal anesthesia.

Methods: This double blind clinical trial was conducted on 40 male patients waiting for elective leg surgery with the age range of 20-60 years in one of the educational hospitals of Kerman University of Medical Sciences, Iran. We used simple random sampling and our participants were assigned into 2 groups (placebo and oral tizanidine receivers). Spinal anesthesia with $1 \mathrm{mg} / \mathrm{kg}$ of hyperbaric lidocaine 5\% was performed in both groups. In tizanidine group, patients received $4 \mathrm{mg}$ of oral tizanidine one hour before spinal anesthesia. Sensory block was examined by pin prick test and all anesthetic duration including start block until reduction of sensory level was calculated at 2 lower dermatomes.

Results: Findings showed that oral tizanidine compared to placebo can cause a 10-15 minute increase in patients' lidocaine spinal anesthesia. Therefore, the average anesthesia time for tizanidine group increased meaningfully $(P=0.03)$. In addition, tizanidine can sedate patients during surgery $(P=0.00)$ or in recovery $(P=0.003)$.

Conclusion: Based on the results, tizanidine increased the duration of lidocaine so oral tizanidine can be used to prolong the duration of lidocaine spinal anesthesia.

Keywords: Tizanidine, Intrathecal lidocaine, Spinal anesthesia
\end{abstract}

\section{Introduction}

Spinal anesthesia is considered as one of the neuraxial blocks which results in (while injecting drug in centroneuraxis) sympathetic block, sensory, and motor block $(1,2)$. Spinal anesthesia is one of the common methods used in surgeries; but, due to time limitations and since anesthetic duration of these drugs is not enough for a surgery to be completed, additive drugs are normally used to increase the duration of surgeries. Vasoconstrictors are one of these additive drugs and the most applicable ones are epinephrine 0.1 and $0.2 \mathrm{mg}$ and phenylephrine $2 \mathrm{mg}(3)$.

These drugs reduce spinal blood flow and subsequently reduce the removal of local anesthetic solution and cause anti-nociception effect by stimulating alpha-adrenergic receivers $(1,3)$. Most of the researchers believe that adding vasoconstrictors to local anesthetic solution can be dangerous especially regarding phenylephrine and epinephrine which are considered strong vasoconstrictors and can cause reduction of spinal blood reception (1). In some studies, increased transient neurologic symptoms have been reported after adding phenylephrine and epinephrine to tetracaine (3). In addition, adding epinephrine in spinal anesthesia can increase lidocaine nervous damages. Also, adding this drug to local anesthetic solution in diabetic patients can increase the risk of ischemic nervous damages (1).

Other drugs added to local anesthetic solutions break acetylcholine, release nitric oxide in spinal cord and thus increase block duration and intensity. Neostigmine is one of the drugs whose application has been questioned due to nausea and lengthened motor block in combination with local anesthetic drugs (1). Adding opioids to local anesthetic drugs increases their effects and decreases pain after operation. However, using these drugs is not common since they delay patient's release from hospital and cause 
the patients to be hospitalized regarding respiratory suppression (3). Adding clonidine to local anesthetic drugs is not as effective as narcotics and at the same time increases hypotension and sympatholytic effects of local anesthetic drugs and lengthens motor blocks $(3,4)$

Like clonidine, tizanidine is an alpha-2-adernorceptor agonist which is mainly used as a relaxant with central effect. Derivatives of clonidine can cause some effects like relaxation, anxiety, and pain reduction; whereas they reduce blood pressure and create less bradycardia. Tizanidine has a direct effect on spinal cord and prevents "simulating amino acids" (NMDA receptors stimulant) from releasing and its preemptive effects have in fact been known (5).

Tizanidine side-effects are mainly related to its dose and include sleepiness, xerostomia, fatigue, weakness, head-ache, bradycardia, arterial blood pressure drop, and digestive and hepatic disorders (6). This drug is available in Iran markets in the form of 2 and $4 \mathrm{mg}$ pills. It is absorbed by digestive system and reaches maximum plasma con-centration within 1-2 hours; and its excreting half-life is $2-4$ hours (7).

Nowadays, performing spinal anesthesia in surgeries limited to lower limbs and pelvis has been taken into huge consideration regarding less side effects, easy performance, patient's satisfaction, and immediate anesthesia. Therefore, to lengthen anesthesia duration and to improve its quality, various drugs have been used. Concerning the benefits and advantages of tizanidine rather than clonidine especially in terms of homodynamic (8) and cardiovascular stability; and regarding the recommendations found in previous studies considering the application of oral tizanidine pills to lengthen neuraxial anesthesia; and due to its relaxation and anti-nociception effects, we decided to examine the effect of 4-miligram Tizanidine pill (taken one hour prior to surgery) on duration of lidocaine spinal anesthesia in a clinical trial.

\section{Methods}

This study was a randomized double-blind, clinical trial. Sampling was simple and 40 male patients waiting for elective leg surgery with the age range of 20-60 entered the study (Class American Society of Anesthetists [ASA] I \& II were selected in one of the educational hospitals of Kerman). These patients were not prohibited from taking tizanidine and performing spinal anesthesia. At first, they were visited and consulted before entering into the surgery room and those who did not sign the consent form were omitted from the study. After determining the necessary criteria, patients were placed in one of the two study groups. Patients with cardiovascular, hepatic, and renal diseases, those who used narcotics and alcohol and those who used anti-hypertension and beta-blocker drugs were excluded. One hour prior to the surgery, patients took oral tizanidine or placebo (in packages similar to original drug) with $30-50 \mathrm{~mL}$ water. After arriving to the surgery room, patients were placed on surgery beds, and then their electrocardiography, pulse oximeter, non-aggressive blood pressure, and sedation levels were monitored and evaluated. All of the patients received $7 \mathrm{cc} / \mathrm{kg}$ crystalloid ringer liquid as inter-vascular compensatory volume. Then in a sitting position, spinal anesthesia was done with $1 \mathrm{mg} / \mathrm{kg}$ hyperbaric lidocaine 5\% using Quincke 23 G Needle in third and fourth or fourth and fifth lumbar apace; all sterilization conditions were observed. Immediately after injection, patients were asked to lie in a supine position and again their electrocardiography, pulse oximeter, blood pressure, heartbeat, and sedation (based on Kulka criterion) were monitored by blinded anesthetist assistance.

\section{Kulka Criterion:}

$0=$ awake

$1=$ sleepy or sedate who awakes easily

$2=$ sleepy who reacts quickly to verbal stimulus

$3=$ sleepy who reacts to verbal stimulus with a short delay $4=$ unconscious

Anesthesia duration was measured (in terms of minute) from anesthesia stability time in the highest sensory dermatome to anesthesia return to two lower dermatomes and block recovery based on reduction of anesthesia surface to four lower dermatomes. Anesthetic surface was examined using pin prick after inter-spinal injection.

If the patient's systolic blood pressure was less than 90 $\mathrm{mm} /$ silver, ephrin drug was used to correct it and in case of nausea, vomit, or heartbeat rate less than $50 \mathrm{pulse} / \mathrm{min}$, $0.6 \mathrm{mg}$ venous atropine was prescribed. After collecting the information, data were recorded in SPSS version 12. To compare data, $t$ test and Mann-Whitney $\mathrm{U}$ test were used. Concerning qualitative variables, chi-square test was used accordingly. Meaningful level was considered to be 0.05 .

\section{Results}

Demographic indexes like age, weight as well as basic blood pressure and heartbeat in both groups revealed no significant differences between these two groups (Table 1). Results showed that oral tizanidine, compared to placebo, can cause a 10-15 minute increase in patients' lidocaine spinal anesthesia so that the average anesthesia time for tizanidine and placebo groups was $57 \pm 16$ and $47 \pm 11$ minutes respectively (increased meaningfully, $P=$ 0.03 ). However, the stability time of spinal anesthesia in tizanidine and placebo was $8 \pm 2.9$ and $7 \pm 2.5$ minutes respectively (no statistically significant difference) (Table 2).

Tizanidine did not cause significant hemodynamic interference unlike placebo group. Blood pressure and heart rate changes in both groups were not related to a special time and no meaningful relationship was found in both groups regarding time of mentioned changes (whether before, during or after operation) (Figures 1 and 2). 
Table 1. Comparison of general indexes in both groups

\begin{tabular}{lccc}
\hline \multirow{2}{*}{ Examined index } & \multicolumn{2}{c}{ Treatment group (mean \pm SD) } & \multirow{2}{*}{$\boldsymbol{P}$ value } \\
\cline { 2 - 3 } & Placebo & Tizanidine & 0.8 \\
\hline Weight $(\mathrm{kg})$ & $68.1 \pm 7$ & $67.7 \pm 5$ & 0.6 \\
Age $(\mathrm{y})$ & $33.1 \pm 12$ & $31.1 \pm 10$ & 1 \\
Basic blood pressure $(\mathrm{mm} \mathrm{Hg})$ & $125.81 \pm 13$ & $127.79 \pm 10$ & 0.09 \\
Average basic blood pressure $(\mathrm{mm} \mathrm{Hg})$ & $95.24 \pm 11$ & $94.97 \pm 12$ & 0.08 \\
Average blood pressure during surgery $(\mathrm{mm} \mathrm{Hg})$ & $84.7 \pm 19$ & $89.3 \pm 17$ & 0.05 \\
\hline Average basic heartbeat (beat/min) & $88.9 \pm 18$ & $85.85 \pm 12$ & \\
\hline
\end{tabular}

Table 2. Comparison of spinal anesthesia in various treatment groups (min)

\begin{tabular}{|c|c|c|c|}
\hline \multirow{2}{*}{ Examined index } & \multicolumn{2}{|c|}{ Treatment group (mean \pm SD) } & \multirow{2}{*}{$P$ value } \\
\hline & Placebo & Tizanidine & \\
\hline Beginning point & $7.2 \pm 2.5$ & $8.0 \pm 2.9$ & 0.35 \\
\hline Reduction of sensory block to 2 dermatomes & $46.5 \pm 11.0$ & $56.6 \pm 16.0$ & 0.03 \\
\hline Reduction of sensory block to 4 dermatomes & $72.4 \pm 14.0$ & $60.7 \pm 13.0$ & 0.01 \\
\hline Recovery duration & $26.5 \pm 11.0$ & $19.6 \pm 9.0$ & 0.05 \\
\hline
\end{tabular}

Another finding of this research is that, tizanidine can sedate patients during surgery $(P=0.00)$ or in recovery $(P=0.003)$; it also decreases the need for using sedation and venous sedatives. These results were statistically very valuable and meaningful (Table 3 ).

No increase was reported in the use of venous liquids,

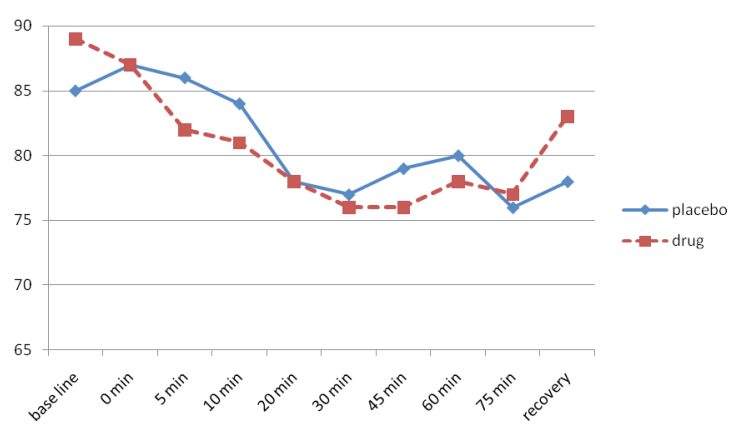

Figure 1. The trend of heartbeat changes (number/minute) in both groups

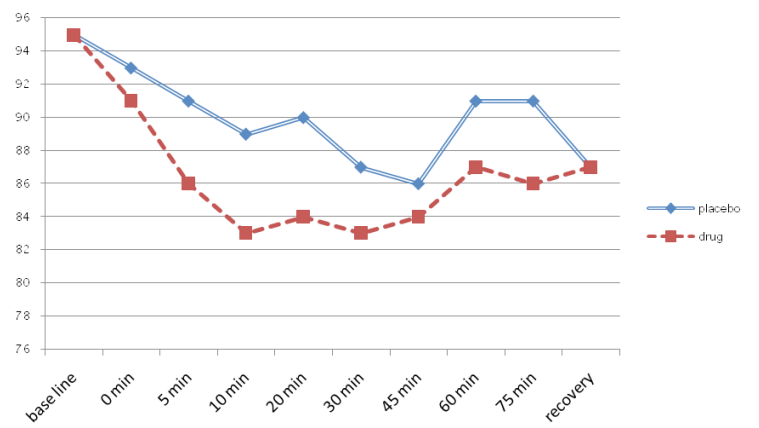

Figure 2. The trend of average blood pressure changes $(\mathrm{mm} \mathrm{Hg})$ in both groups. atropine and ephedrine in those who had used tizanidine. None of the patients in this study showed a special problem during anesthesia or after it.

We also evaluated the side effects of drug (e.g. bradycardia, nausea, reduced blood pressure, headache, and sleepiness) in both groups. We did not observe a significant difference between groups.

\section{Discussion}

Results of this research showed that the consumption of oral tizanidine one hour prior to surgery can lengthen lidocaine spinal anesthesia. Having tranquilizing and sedative effects, this drug reduced patients' fear and anxiety during operation and increased their relaxation during surgery and recovery and thus decreased the consumption of venous tranquilizers. Moreover, using tizanidine in lidocaine spinal anesthesia did not cause any changes in needs to atropine, ephedrine, and venous liquids.

Spinal anesthesia is one of the common methods used in surgeries; however, due to time limitations and since anesthetic duration of these drugs is not enough for a surgery to be completed, additive drugs are normally used along with them to increase the duration of surgeries. However, using these drugs in inter-spinal injections may cause cardiovascular problems $(1,2)$.

In a study carried out in 1995 in Japan on gynecologic patients who had tetracaine spinal anesthesia, the effects of oral tizanidine and clonidine were compared. The results showed that both drugs could lengthen tetracaine spinal anesthesia while tizanidine had lower side effects and caused better hemodynamic stability (8). In this study, tizanidine increased lidocaine's effect and did not cause homodynamic effects.

In another research which was done in 2008, inter- 
Table 3. Comparison of variables frequency (class ASA, sedation, atropine and ephedrine consumption)

\begin{tabular}{|c|c|c|c|c|c|}
\hline \multirow{2}{*}{ Variable } & \multicolumn{2}{|c|}{ Placebo $(n=20)$} & \multicolumn{2}{|c|}{ Drug $(n=20)$} & \multirow{2}{*}{$\begin{array}{c}P \\
\text { value }\end{array}$} \\
\hline & No. & $\%$ & No. & $\%$ & \\
\hline \multicolumn{6}{|l|}{ Class ASA } \\
\hline I & 18 & 90 & 18 & 90 & 1 \\
\hline II & 2 & 10 & 2 & 10 & 1 \\
\hline Sedation before surgery & 0 & 0 & 4 & 20 & 0.1 \\
\hline Sedation during surgery & 1 & 5 & 15 & 75 & 0 \\
\hline Sedation in recovery & 0 & 0 & 8 & 40 & 0.003 \\
\hline Ephedrine consumption & 1 & 5 & 2 & 10 & 1 \\
\hline Atropine consumption & 4 & 20 & 3 & 15 & 1 \\
\hline
\end{tabular}

spinal lidocaine was added to local anesthetic drugs. The findings showed an increase in arterial hypotension episodes but no bradycardia was observed (4). In this research, bradycardia and arterial hypotension were not found in patients.

Asgari et al investigated the effect of subdiaphragmatic infiltration of lidocaine $1 \%$ at the beginning of surgery combined with spinal anesthesia in order to reduce pain, but contrary to prior studies, their results showed that mean severity of pain among patients was not significant and adding lidocaine $1 \%$ did not affect severity of pain. In the present study, spinal anesthesia was done by $1 \mathrm{mg} / \mathrm{kg}$ of hyperbaric lidocaine $5 \%$ (9).

In a study carried out by Gabriel and Gordin in 2001 in the United States, clonidine along with local anesthetic drugs increased sensory block regardless of starting point (5). In our study, starting point was not influenced by tizanidine, and anesthesia duration increased.

In another research performed by Miettinen et al, sedative effects of $12 \mathrm{mg}$ oral tizanidine was comparable with $50 \mu \mathrm{g}$ clonidine but clonidine effects were longer; but, diastolic blood pressure drop in clonidine was higher than that of tizanidine. In this research, oral tizanidine was introduced as a suitable substitute for clonidine during operation (7). Rupani et al indicated that preoperative oral tizanidine improved the severity of postoperative analgesia without increasing side effect. Their results are along with the present stud (10).

A study conducted by Shoeibi et al showed that prescription of oral tizanidine as premedication patients with spinal anesthesia in order to reduction the pain arises from needle puncture was significant and increases satisfaction of anesthesia due to prepared analgesia. Furthermore, the mean of heart rate and diastolic blood pressure in patients treated with tizanidine was lower than placebo group (11). Talakoub et al remarked that administration of tizanidine before operation in patients scheduled for elective laparoscopic cholecystectomy under general anesthesia improves postoperative analgesia, provides less pain intensity and decreases the amount of analgesic consumption (12).

Henney et al carried out their study about pediatric safety of tizanidine. They implied that the overall safety of tizanidine in the pediatric group was good. None of the patients in the present study had a special problem during anesthesia or after it and we did not observe significant differences between the two groups concerning the side effects of the drug (13).

In this research, the side effects of tizanidine such as hypotension, bradycardia, nausea, headache, and sleepiness in placebo and tizanidine groups were not significantly different, while patients' relaxation was higher based on Kulka criterion.

\section{Conclusion}

In this study, the effect of oral tizanidine on the duration of lidocaine in spinal anesthesia was examined. It was shown that tizanidine increased the duration of lidocaine more than placebo; homodynamic change in both groups was not significant. It is suggested that various doses of tizanidine and various times of prescription prior to operation should be examined.

\section{Ethical issues}

The written informed consent was obtained from participants. This study was approved by Kerman University of Medical Sciences ethics committee with ethics code: IR.KMU.REC.1396.19.

\section{Authors' contributions}

All authors contributed to drafting/revising the manuscript as well as study concept or design, analysis or interpretation of data.

\section{References}

1. Brown DL. Spinal, epidural and caudal anesthesia. In: Anesthesia. 5th ed. Philadelphia, Pa: Churchill Livingstone; 2000:1510.

2. Montazeri K, Ghobadin AH. Effect of oral clonidine as premedication on duration OF block hemodynamic changes and need of ephedrine on spinal anesthesia. Journal of Iranian Society of Anesthesiology \& Intensive Care 2004; 25: 75-6.

3. Drasner K, Larson M. Spinal and epidural anesthesia. In: Stoelting RK, Miller RD, eds. Basics of Anesthesia. 5th ed. Philadelphia: Churchill Livingstone; 2007. p. 241-71.

4. Elia N, Culebras X, Mazza C, Schiffer E, Tramer MR. Clonidine as an adjuvant to intrathecal local anesthetics for surgery: systematic review of randomized trials. Reg Anesth Pain Med 2008; 33(2): 159-67. doi: 10.1016/j. rapm.2007.10.008.

5. Gabriel JS, Gordin V. Alpha 2 agonists in regional anesthesia and analgesia. Curr Opin Anaesthesiol 2001; 14(6): 751-3. doi: 10.1097/00001503-200112000-00024.

6. Imanaga $\mathrm{K}$, Wajima Z, Inoue $\mathrm{T}$, Ogawa R. Effect of oral tizanidine on local-anesthetic infiltration pain during epidural catheterization. J Nippon Med Sch 2004; 71(2): 105-10. doi: 10.1272/jnms.71.105.

7. Miettinen TJ, Kanto JH, Salonen MA, Scheinin M. The sedative and sympatholytic effects of oral tizanidine in healthy volunteers. Anesth Analg 1996; 82(4): 817-20. doi: 
10.1213/00000539-199604000-00024.

8. Omote K, Satoh O, Sonoda H, Kumeta Y, Yamaya K, Namiki A. [Effects of oral alpha 2 adrenergic agonists, clonidine and tizanidine, on tetracaine spinal anesthesia]. Masui 1995; 44(6): 816-23. [In Japanese].

9. Asgari Z, Rezaeinejad M, Hosseini R, Nataj M, Razavi M, Sepidarkish M. Spinal Anesthesia and Spinal Anesthesia with Subdiaphragmatic Lidocaine in Shoulder Pain Reduction for Gynecological Laparoscopic Surgery: A Randomized Clinical Trial. Pain Res Manag 2017; 2017: 1721460. doi: 10.1155/2017/1721460.

10. Rupani M, Alijanpour E, Banihashem N, Alipour A, Shirkhani Z, Solimanian S. Effect of Oral Tizanidine on Postoperative Pain after Perianal Surgery. J Babol Univ Med
Sci 2013; 15(4): 7-11. doi: 10.18869/acadpub.jbums.15.4.7.

11. Shoeibi G, Soltani Mohammadi S, Ahangari A, Marashi S, Borji A, Yaghooti AA. Effects of Oral Tizanidine Premedication on Pain and Hemodynamic Responses after Spinal Needle Skin Puncture. Archives of Anesthesiology and Critical Care 2015; 1(1): 18-20.

12. Talakoub R, Abbasi S, Maghami E, Zavareh SM. The effect of oral tizanidine on postoperative pain relief after elective laparoscopic cholecystectomy. Adv Biomed Res 2016; 5: 19. doi: 10.4103/2277-9175.175905.

13. Henney HR 3rd, Chez M. Pediatric safety of tizanidine: clinical adverse event database and retrospective chart assessment. Paediatr Drugs 2009; 11(6): 397-406. doi: 10.2165/11316090-000000000-00000. 